\title{
EL DESASTRE DESDE LOS INDIVIDUOS: LOS CASOS DE PELLUHUE Y CONSTITUCION EN CHILE
}

Francisco Javier Jorquera Santis ${ }^{1, *}$

\section{RESUMEN}

El presente trabajo tiene como objetivo conocer los significados que el desastre del 27 de febrero de 2010 en Chile tuvo en las trayectorias de vidas de los individuos de las comunas de Constitución y Pelluhue, y describir las acciones colectivas que desplegaron ambas comunidades en función de responder y afrontar el incidente crítico. Además, se explora la forma en que el Estado, la Comunidad y su cultura influyeron en las estrategias desplegadas y en las significaciones elaboradas por los individuos protagonistas, entendiendo dicha articulación como factor de vulnerabilidad. Dichos objetivos se plantean con el propósito de contribuir al desarrollo de estrategias locales orientadas a la reducción del riesgo de desastres. La investigación es de carácter social cualitativa basada en narrativas, y considera como sujetos de estudio a quienes vivieron la experiencia en ambas comunas. Los resultados describen el comportamiento del colectivo en ambas localidades durante la respuesta y recuperación, y concluye que fueron condicionados por factores de vulnerabilidad socioeconómicos, político-institucionales y psicosociales, incidiendo además en la capacidad de resiliencia de dichas comunidades. También se destaca la promoción del individuo como actor social capaz de transformar su realidad social.

\section{PALABRAS CLAVE}

Desastre, Significado, Individuo, Comunidad, Resiliencia

\section{DISASTER FROM INDIVIDUALS: THE CASES OF PELLUHUE AND CONSTITUCION IN CHILE}

\section{ABSTRACT}

This work investigates the meanings that the disaster of February 27, 2010 in Chile had in the trajectories of lives of the individuals in the localities of Constitución and Pelluhue. Likewise, it seeks to describe the collective actions that both communities deployed in order to respond to and recover from the disaster. In addition, the way in which the national government and the State, communities and their culture influenced and articulate the strategies deployed and the meanings elaborated by the protagonists is explored. With this, the work tries to understand such articulation as a factor of vulnerability. All these objectives are set with the purpose of contributing to the development of local strategies for disaster risk reduction. The research adopts a social and qualitative approach based on narratives, considering as subjects of study those who lived the disaster experience in both communities. The results describe the behavior of groups in both locations during the response and recovery, and allow to conclude that they were conditioned by socioeconomic, political-institutional and psychosocial vulnerability factors, also affecting the resilience of these communities. It also highlights the promotion of the individual as a social actor capable of transforming their social reality.

\section{KEYWORDS}

Disaster, Meaning, Individual, Community, Resilience
1. Corporación Gestión de Riesgos y Desastres GRID Chile, Santiago, Chile.

*Autor de correspondencia: fjorquerasantis@gmail.com

\section{RECIBIDO}

28 de marzo de 2019

\section{ACEPTADO}

22 de mayo de 2019

\section{PUBLICADO}

1 de julio de 2019

\section{Formato cita}

Recomendada (APA): Jorquera Santis, F.J. (2019). El desastre desde los individuos: los casos de Pelluhue y Constitucion en Chile. Revista de Estudios Latinoamericanos sobre Reducción del Riesgo de Desastres REDER, 3(2), 58-70.

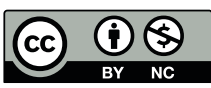

Todos los artículos publicados en REDER siguen una política de Acceso Abierto y se respaldan en una Licencia CreativeCommons Atribución-NoComercial 4.0 Internacional.

Revista de Estudios

Latinoamericanos sobre Reducción del Riesgo de Desastres (REDER)

Diseño: Lupe Bezzina Tipografía: Hospital 


\section{INTRODUCCIÓN}

Una serie de estudios se han llevado a cabo durante las últimas tres décadas con el fin de conceptualizar y gestionar los riesgos de desastres aumentando la capacidad de preparación, mitigación, prevención, respuesta y recuperación de los Estados y las comunidades, comprendiendo que el riesgo de un desastre corresponde a una construcción social (Narváez, Lawell, Pérez, 2009), y no a un fenómeno netamente natural. En ese sentido se entiende que los riesgos se constituyen en base a la relación dialógica entre las vulnerabilidades o los factores de vulnerabilidad y las amenazas, las cuales pueden ser naturales o antropogénicas. La vulnerabilidad ante el riesgo de desastres corresponde a las probabilidades de que un individuo o un grupo de ellos, esté expuesto a una amenaza, sea ésta de origen natural o antropogénica (Chardon, 2008). Ésta es generada por diversos factores asociados a procesos físico-naturales, socioeconómicos, técnicos, políticosinstitucionales y funcionales (Chardon, 2008). Esto significa que el concepto de vulnerabilidad se dinamiza al tener que considerarse durante todo el proceso del desastre, o sea antes, durante y después del incidente crítico. Por ende la vulnerabilidad también implica ineficacia que pueden tener los Estados y las comunidades tanto como para anticipar los efectos de un desastre, su capacidad de resistir a éste y la generación de resiliencia a raíz de la recuperación. Sumado a ello, las capacidades las conceptualizamos como el conjunto de fortalezas y recursos de una sociedad o de una comunidad, para reducir el nivel del riesgo, responder de forma efectiva y generar resiliencia y capital social en el proceso de recuperación post incidente crítico de un desastre (Romero \& Vidal, 2015).

Por lo tanto, la reducción de los factores de vulnerabilidad y el aumento de las capacidades en torno a la resiliencia y el capital social, son claves para la reducción del riesgo de desastre, en tanto gestión del riesgo de desastres como política pública y como estrategias locales a desarrollar. En ese sentido, resalta la importancia que tienen los individuos para la reducción del riesgo de desastres, en el sentido de su rol como actores sociales capaces de generar acciones comunitarias, sociales y políticas para dicho cometido, generando conocimiento local a raíz de las experiencias vividas en torno a cada desastre. Por lo tanto, este trabajo busca conocer los significados y el sentido que tienen los desastres en las trayectorias de vidas de los individuos, conocer las estrategias de afrontamiento desplegadas y conocer su capacidad de resiliencia, para desde allí desarrollar estrategias en torno a reducir la vulnerabilidad, desde una mirada comunitaria, local y territorial, considerando las características propias de un territorio determinado, sea un barrio o un sector 0 una comuna o un conjunto de éstas.

Además, este estudio se suma a un conjunto de investigaciones sociales realizadas previamente en torno al desastre del 27 de febrero de 2010 en Chile o 27F (Baeza, 2010; Dresdner \& Sehnbruch, 2010; Rojas, 2010; Valenzuela, 2010; Salgado \& Ugarte, 2014).

\section{CONTEXTO}

El Chile contemporáneo es producto y en tanto proceso de la fase tardía del Capitalismo Neoliberal desarrollado en los últimos cuarenta años, en la fase en donde tanto el Estado como el Mercado compiten en cada ámbito de la experiencia social de los individuos (Ruiz \& Boccardo, 2014). Considerando que el Neoliberalismo o Capitalismo Neoliberal es más un momento histórico que un modelo de sociedad (Araujo \& Martuccelli, 2012), existen en los contextos de desastres acontecimientos extraordinarios (Goffman, 1974) en donde la narrativa hegemónica del yo hiperindividualizado contiene narrativas subalternas sobre un nosotros comunitario, similar a las narrativas que podemos visualizar y describir en otros contextos coyunturales, como lo son los movimientos sociales, especialmente en el caso del movimiento estudiantil o los movimientos ecologistas. No obstante, el individualismo, la competitividad y el consumo, permean en los procesos de individuación de los habitantes de Chile, incluso en aquellos contextos rurales en donde estos atisbos de modernización se mezclan con características típicas de la Hacienda, singularidad que podemos encontrar en la zona central donde ocurrió el desastre del 27F. Por otro lado el Estado ha generado un conjunto de mecanismos mediante los cuales, desde la condición biológica de la especie humana frente a los desastres, contiene una estrategia o política de control de los individuos, como una estrategia de poder, una forma de biopoder (Foucault, 2004). Por lo tanto el Estado a través de su política de protección civil y su política de seguridad pública ejerce su poder sobre los individuos en tanto protege a la ciudadanía de las inclemencias de la naturaleza y de los comportamientos desviados que ocurren en el momento de mayor crisis, normando el comportamiento para afrontar el incidente y su posterior recuperación, en tanto corresponden al desarrollo de tecnologías de 
biopoder. O sea "el Estado que garantiza la seguridad es un Estado que está obligado a intervenir en todos los casos en los que la trama de la vida cotidiana es agujereada por un acontecimiento singular, excepcional" (Foucault, 2004).

La Sociología de la Individuación nos permite reflexionar sobre los procesos de individuación que se centran, desde una perspectiva socio-histórica, en el tipo de individuo que es estructuralmente fabricado en una sociedad particular (Araujo \& Martuccelli, 2012). Por lo que la individuación es una perspectiva particular de análisis que se interroga por el tipo de individuo que es estructuralmente producido por una sociedad en un período histórico determinado. Para dar cuenta de ello es preciso privilegiar exclusivamente algunos grandes factores estructurales, los cuales se conceptualizan como pruebas societales. Las pruebas son en este sentido desafíos históricos, socialmente producidos, culturalmente representados, desigualmente distribuidos que los individuos están obligados a enfrentar en el seno de un proceso estructural de individuación (Araujo y Martuccelli, 2012). Estas poseen ciertas características analíticas: Una dimensión narrativa; suponen un efecto desde donde los actores experimentan y comprenden sus vidas sometidas a un conjunto de problemas específicos. Las narrativas de los sujetos dan cuenta de una trayectoria de vida como una sucesión permanente de puestas a prueba. En el caso del desastre, es que éste se significa como un desafío en el sentido de acentuarse un conjunto importante de estas pruebas que los sujetos deben sortear y superar en su cotidianeidad, convirtiendo a estas pruebas en factores de vulnerabilidad ante el riesgo de desastre. Por otro lado, las pruebas suponen un tipo de individuo que se encuentra obligado, debido a razones estructurales, a enfrentar cada uno de estos desafíos (Araujo y Martuccelli, 2012).

Comprendiendo lo anterior, es necesario conocer cómo los individuos le dan significado y sentido a su experiencia de afrontar el desastre, y cómo las estrategias desplegadas son influenciadas y a veces condicionadas por la articulación de ciertos factores de riesgo de vulnerabilidad ante desastres, especialmente aquellos político-institucionales, psicosociales y socioeconómicos, los cuales generalmente se presentan como pruebas societales en la cotidianeidad de los sujetos. Esta dimensión subjetiva de la experiencia en desastres es importante para la generación de estrategias locales de reducción del riesgo de desastres, sustentadas en un conocimiento situado a nivel local.

Por lo tanto el presente trabajo tiene como objetivo conocer los significados del $27 \mathrm{~F}$ en las comunas de Constitución y Pelluhue en Chile, y describir las acciones colectivas que desplegaron ambas comunidades en función de responder y afrontar el incidente crítico, considerando la articulación de factores de riesgo político-institucionales, factores de riesgo socioeconómicos y factores de riesgo psicosociales. Esto entendiendo el desastre socionatural y el comportamiento humano en éste como fenómeno social, en tanto los fenómenos sociales se desarrollan particularmente desde contextos sociales específicos en el tiempo y en el espacio.

\section{F: LOS CASOS DE CONSTITUCIÓN Y PELLUHUE}

Constitución corresponde a una comuna costera de la Región del Maule, Chile. Cuenta con una superficie de $1.343,6 \mathrm{~km}^{2}$ y una población de 46.068 habitantes (Censo INE, 2017), aglomerando la pequeña ciudad del mismo nombre y varias localidades pesqueras y localidades campestres en medio de la cordillera de la costa, completamente rurales. Su principal fuente económica es producida por la Celulosa Arauco y Constitución (CELCO) orientada a la producción de papel. En el 2010 fue una de las comunas más afectadas por el desastre, especialmente la pequeña ciudad la cual fue afectada tanto por el terremoto como por el maremoto. Esa noche, algunos pescadores se preparaban para salir en la madrugada al mar y un número importante de habitantes se encontraba en la popular Isla Orrego, en medio del Río Maule, celebrando la noche veneciana, típica fiesta de fines de verano de la ciudad.

La Comuna de Pelluhue está ubicada en la costa sur de la Región del Maule, siendo parte de la Provincia de Cauquenes. Está compuesta por dos pueblos: Pelluhue y Curanipe, siendo en la segunda en donde se encuentra la municipalidad. Además contiene otras localidades rurales, tanto costeras como de la cordillera de la costa, como lo son Mariscadero y Chovellén. Pelluhue a nivel comunal, además abarca una superficie de $371,4 \mathrm{~km} 2$ y tiene una población de 7.571 habitantes (Censo, 2017). El 2010 fue una de las comunas más afectadas por el terremoto y maremoto, siendo afectada principalmente la localidad de Mariscadero, en el extremo norte de la comuna.

El terremoto ocurrió a las 03:34:08 hora local (UTC-3), del sábado 27 de febrero de 2010, alcanzando una magnitud de 8,8 MW (U.S. Geological Survey, 2010), con epicentro en el mar 
chileno, frente a las localidades de Curanipe y Cobquecura, cerca de 150 kilómetros al noroeste de Concepción y a 63 kilómetros al suroeste de Cauquenes, y a 30,1 kilómetros de profundidad bajo la corteza terrestre. Las víctimas fatales llegaron a 525 personas, cerca de 500 mil viviendas damnificadas, y un total aproximado de 2 millones de damnificados.

\section{ARTICULACION DE SIGNIFICADOS}

Con respecto a los significados, Bruner (1991) enfatiza en la activa construcción de significados de las personas, quienes basan sus actos en intenciones, creencias, expectativas, esperanzas y valores, y hace alusión al concepto de psicología popular o psicología cultural, la cual refiere a cómo en los contextos culturales se constituyen discursos canónicos sobre "lo que es" y "lo que debe ser", emergiendo narrativas que organizan y le dan significado a la experiencia. Por lo tanto, la experiencia y su respectiva significación tienen un carácter eminentemente social. El énfasis y la importancia queda puesta de esta manera en el rol de la cultura en la construcción, mantención y re-construcción constante de los significados. A su vez, Geertz (1983) define la cultura como aquella dimensión o realidad a la cual debemos adaptarnos recursivamente, de donde obtenemos las herramientas para poder distinguirnos como individuos y donde creamos un mundo de significados. Es la fuente de "sentidos" con que damos significados a los fenómenos de la vida cotidiana, para poder interactuar socialmente. En ese sentido, las perspectivas de la acción situada (Sandoval, 2004), entendidas como "una visión epistemológica desde la cual todas las formas de conocer de nuestra vida social están situadas en un contexto histórico y corporal" (Sandoval, 2013, p.38), los procesos de significación y construcción social de la realidad, primero "deben ser considerados como acciones situadas en un trasfondo de naturaleza semiótico-material sedimentado como corporalidad y forma de vida; y segundo que el proceso de construcción de la realidad no corresponde a una acción unilateralmente humana, sino que más bien responde a un proceso de articulación e hibridación entre agencias de naturaleza material y simbólica" (Sandoval, 2013, p.38). Dicho trasfondo corresponde a un contexto de tradiciones, de relaciones de poder, de creencias, de saberes y prácticas primeramente biológicas y necesariamente sociales. Bourdieu afirma: "El cuerpo cree en lo que juega: Ilora cuando mima la tristeza. No representa lo que juega, no memoriza el pasado, actúa el pasado, anulado así en tanto que tal, lo revive" (Bourdieu, 1991, p.124). Por lo tanto la idea de trasfondo da cuenta de su base corporal y performativa de la acción, entendiendo por tanto el cuerpo como un contexto de sentido de nuestras experiencias, y también de la propia investigación. Además, en el mundo co-existen muchos actores, no todos humanos, ni todos orgánicos, ni todos tecnológicos (Haraway, 1995), por lo que el concepto de articulación invita a entender "el proceso de constitución del mundo desde la coordinación, traducción, conflicto y mestizaje que supone que algo se "articule" con otra cosa" (Sandoval, 2013, p.39), concepto cuya emergencia y uso proviene de diferentes autores como Latour, Haraway, Laclau y Mouffe (Sandoval 2004). A través de este concepto se logra analizar como los individuos articulan el significado del desastre considerando otros actores y escenarios cargados de significado como lo son, el mar, la tierra, los bosques, las industrias, los animales, las deidades, entre otros. Siguiendo la misma línea, los conocimientos son situados (Haraway, 1995; Sandoval, 2004), en tanto parciales y posicionados en trasfondos semiótico-materiales de las articulaciones en constante transformación, posibilitando el conocimiento siempre "desde un cuerpo, un tiempo y un lugar" (Sandoval, 2004, p.37).

Además es pertinente considerar dos premisas fundamentales: Primero, que los seres humanos otorgamos sentido a nuestras experiencias pensándolas como historias, relatos o narraciones (Ricoeur, 2008), siendo además, relatar, una forma de comunicación, a través de lo lingüístico y extralingüistico. Segundo, que el hecho de narrar nos permite organizar acciones y actores (humanos y no humanos) en torno a un significado, estructurando nuestra experiencia del tiempo, conectándolas con las metanarrativas cristalizadas o sedimentadas en el trasfondo semiótico-material que a su vez condicionan nuestro deber ser. Por ende, las narrativas se conciben "como una forma fenomenológica y epistemológica de la comprensión y de expresión" (Riessman, 2008).

\section{METODOLOGÍA}

El presente trabajo tiene un carácter exploratorio y descriptivo. Exploratorio pues aborda una dimensión con escasas referencias de investigaciones anteriores en las dos poblaciones desarrollada. Descriptivo, en el sentido que describe y detalla los relatos de las/los sobrevivientes al desastre, dando cuenta de sus significados en torno a dicha experiencia. Por otro lado, el trabajo tiene un carácter transversal puesto que el proceso de producción de datos ha sido en un momento del 
continuo de las trayectorias de vidas de las/los sujetos de investigación. Se utilizó una metodología narrativa como un tipo de metodología cualitativa que tiene por objetivo principalmente, la captación y reconstrucción de significado (Ruiz, 2003), que produce datos descriptivos provenientes de las propias palabras de las personas, habladas o escritas, y de la conducta observable desde el paradigma interpretativo-hermenéutico (Bogdan y Taylor 1986). La producción de información se realizó a través de entrevistas narrativas (Riessman, 2001; Biglia \& Jordi Bonet-Martí, 2009). Usando esta técnica se produjeron diecisiete narrativas. El análisis se realizó desde los métodos narrativos empleados en las ciencias sociales (Riessman, 2008; Bernasconi, 2011).

La entrevista narrativa consistió en la reconstrucción realizada por el/la entrevistados/a de una parte de su vida, a través de una línea basada en la sucesión de hechos y situaciones desde el incidente crítico hasta el período en que se realizaron las entrevistas. No obstante se incluyeron preguntas sobre conocimientos previos, en forma de flash backs y profundizaciones en algunos momentos focalizados en las estrategias de afrontamiento. En tanto se realizaron las entrevistas, constantemente se consultó al/a la entrevistado/a por las propias interpretaciones del investigador, con respecto a su experiencia, sus acciones y las intenciones subyacentes a éstas, así como los sentidos de sus experiencias y acciones concretas. Esto, en base a las Producciones Narrativas (PN) de Balasch y Montenegro (2003), basada en la co-escritura de narrativas entre investigador/a y entrevistado/a, similar además a la propuesta de entrevista narrativa de Martin Bauer (1996). Cabe destacar que las conversaciones no abordaron aspectos traumáticos de dicha experiencia, focalizándose profundamente en las estrategias individuales y colectivas de afrontamiento, de respuesta y de recuperación.

La muestra fue no probabilística de sujetos tipo. La cantidad y cualidades de los registros que configuraron esta muestra fueron conmensurados según la posibilidad de dar cuenta del objeto de investigación construido (Cottet, 2013). Por lo tanto, la muestra es de representación estructural con el propósito de "representar una red de relaciones, de modo que cada participante puede entenderse como una posición, en una estructura [...] la muestra así tiene la misma forma que su colectivo representado." (Canales, 2006).

\section{INDIVIDUOS, SIGNIFICADOS Y ESTRATEGIAS}

A continuación se exponen los principales hallazgos y conclusiones a raíz de la investigación:

\section{Desastre como punto de inflexión}

El desastre es concebido como un punto de inflexión en la trama de la narrativa identitaria de cada uno de los sujetos que lo experimentaron de forma directa, la cual se articula con el conjunto de narrativas locales. Toma el sentido de un desafío al cual superar, desde el incidente crítico en adelante. A su vez, éste se articula en función de distintas agencialidades: La Tierra comunica su malestar con la acción humana a través del terremoto y tsunami, en tanto Dios quien también castiga, se apiada y protege a la mayoría de los humanos, ante el desamparo del Estado concebido previamente como protector. Esta articulación de agencialidades podría explicarse a raíz de la matriz de creencias judaico-cristiana hegemónica en el mundo rural de la zona central chilena, una forma de secularización inacabada.

Este punto de inflexión no solo implica el abandono de expectativas, planes y anhelos que los sujetos concebían previo al evento, los cuales resignifican para darle sentido, sino que también significan un desafío, el cual está relacionado con dos aspectos importantes a considerar:

1. Siguiendo la idea de pruebas societales (Araujo \& Martuccelli, 2012), en el caso del desastre, significado como un desafío en el sentido de acentuarse un conjunto importante de estas pruebas societales que los individuos deben experimentar en su cotidianeidad, convirtiendo a vez, estas pruebas en factores de vulnerabilidad ante el riesgo de desastre. 0 propuesto de otra forma, estos factores implican las pruebas en la cotidianeidad de los individuos, lo que refuerza el concepto de desastre socionatural.

2. Basado en la idea de los factores de vulnerabilidad ante el riesgo de desastre (Chardon, 2008), se identifican en las narrativas de los individuos factores político-institucionales, factores socioeconómicos (especialmente de clase social), y factores psicosociales (capital social), que se articulan como trasfondo desde donde se significa, y se le da sentido al desastre en las narrativas identitarias de los sujetos. 
El sentido del desastre para la mayoría de los sujetos tiene un carácter inminentemente natural, en desmedro de los factores de vulnerabilidad políticos, económicos y psicosociales, lo que conlleva a la naturalización de dichos factores de vulnerabilidad y por lo tanto manteniendo el canon hegemónico de acciones frente a éstos mismos. Este carácter inminentemente natural surge del sincretismo cultural propio de la zona costera de la Región del Maule.

Por otro lado, el desastre significa una oportunidad de desarrollo para los individuos que pueden maximizar directamente su capital tanto político como económico, por lo que, en algunos casos, la posición dentro de la estructura de clases condiciona la forma en que los sujetos significan y le dan sentido al desastre.

\section{Narrativas}

En relación a las narrativas generadas en las entrevistas, éstas cumplen dos propósitos además de dar respuesta a las preguntas insertas en el diálogo entrevistador-entrevistada/o: Por un lado, son testimonio de toda la experiencia vivida, en tanto cumplen el propósito de denuncia, con respecto al desamparo de las instituciones del Estado. A su vez, fueron narradas en primera persona al ser protagonistas de éstas. Como personajes secundarios se presenta el grupo primario de apoyo, especialmente la familia nuclear. Como personajes episódicos, y con una agencialidad destacada, figuran Dios, la Tierra y el Estado.

La trama de cada historia obedece al género de la tragedia, en donde cada uno encarna el héroe solitario/a, hiperindividualizado por el contexto social desde donde emerge. En cada trama, el o la protagonista sortea los obstáculos dentro del contexto del desastre, siendo una acentuación de las condiciones socioeconómicas presentes antes del incidente crítico. Al respecto, la desigualdad frente a las oportunidades de desarrollo social y económico se refleja en el contexto del desastre, sea a través de las estrategias de afrontamiento desplegadas, las capacidades instaladas previamente, donde el nivel educacional y el nivel socioeconómico, evidenciándose como factores de vulnerabilidad, preponderantes.

Por otra parte, en cada narrativa se evidencia la articulación de conocimientos científicoformales con los conocimientos locales sobre los terremotos y tsunamis, éstos últimos transmitidos oralmente de generación en generación, como forma de memoria viva, al mismo tiempo que se generan nuevos conocimientos con respecto a la toma de conciencia alrededor de la preparación y prevención frente a futuros fenómenos similares. Estos nuevos aprendizajes son principalmente individuales en torno al concepto de autocuidado, como acciones individuales y familiares en la preparación frente a futuros terremotos y tsunamis, siendo la necesidad de generar estrategias de prevención comunitaria más un sentimiento de nostalgia que una acción concreta a desarrollar.

\section{Estrategias}

Las estrategias desarrolladas durante la respuesta al incidente crítico estuvieron enfocadas primeramente a la sobrevivencia, en la evacuación a zonas seguras. Dicho aprendizaje estaba internalizado principalmente en los residentes de ambas localidades, no así en el caso de los turistas. Diferencia que quedó en evidencia, pues a pesar de que las autoridades y los medios de comunicación masiva informaban que no existía alerta de tsunami, los residentes evacuaron a los cerros más próximos. El conocimiento previo fue adquirido a través de la oralidad de la familia y por otro lado, a través del sistema educacional. Por lo tanto se evidencia el impacto que tienen los simulacros escolares en la posterior conducta de los sujetos durante un terremoto y un eventual tsunami. En todas las narrativas se evidencia además la expectativa de la acción del Estado a través de la presencia de autoridades y la alerta de tsunami. Aquí algunos ejemplos.

"ya llevaban hartas veces haciendo [...] ¿cómo se llama? [...] sacar a los niños pa allá [...] simulacro".

(Hombre, 54 años, pescador, La Poza)

"[...] me acuerdo que siempre hacíamos plan Daisy cuando chicos y hasta el día de hoy, o sea, o sea sabemos dónde hay que arrancar, las vías de evacuación, no hay para qué poner letrero, nada, porque la gente sabe dónde tiene que ir".

(Mujer, dirigente social, 37 años) 
"O sea a pesar de que aquí si había planes de esto [...] de los niños que se les hacía tsuna [...] o sea de, ejercicios de alerta, pero no había sido la gente tan informada, sobre todo los que estaban de afuera, porque no había ninguna cosa preventiva".

(Hombre, 73 años, funcionario público y empresario, Chovellén)

"la radio Bíobío que esa noche se escuchaba allá en el campo, informó que estaría todo, que hay que quedarse tranquilo, nos creímos a eso y al día siguiente supimos de lo que había pasado realmente".

(Hombre, 68 años, empresario y político. Curanipe, Comuna de Pelluhue)

Durante el desarrollo de la respuesta ante el terremoto y el tsunami, se describen las siguientes acciones desplegadas:

Heroísmo: Conjunto de acciones que realizó un sujeto o un grupo de ellos con el fin de salvaguardar la vida de otro/s sujetos, sean estos familiares, amigos/as, vecinos/as o desconocidos.

"ahí le dije yo; "oye vamos a ver los botes, si podemos sacar esa gente de ahí de la isla poh"”. Qué es lo que pasó, lo siguiente que cuando el río, el río se chupó porque la mar se recogió, y al recogerse la mar el río se chupó".

(Hombre, 60 años, botero y sindicalista, La Poza).

Solidaridad Comunitaria: Conjunto de acciones colectivas que desplegaron grupos de sujetos a nivel familiar y/o a nivel comunitario para afrontar el incidente crítico en su primer momento: Campamentos improvisados, las ollas comunes levantadas por mujeres principalmente jefas de hogar, grupos de hombres removiendo escombros o recuperando cosas de utilidad entre los escombros, la búsqueda de seres significativos, organización para el acceso a alimentos y útiles de aseo que provenían de otros pueblos. Estas acciones las podemos identificar como parte de las estrategias organizativas, en donde "frente a la ausencia de respuesta de las autoridades ante los problemas concretos, parte de la población recurrió a modalidades de acción individuales o colectivas, para cubrir las necesidades y expectativas de la comunidad, (Arteaga \& Pérez et al., 2015, p.8). En otras palabras acciones desplegadas para enfrentar la crisis de forma comunitaria. Por lo que se puede afirmar que "ciertos elementos más arraigados de una cultura chilena, confrontada a catástrofes de manera periódica, que vinculan a la solidaridad como valor social por ejemplo, tienden a reaparecer en la superficie" (Baeza, 2010, p.67). En el caso de las acciones desplegadas en la fase de respuesta el sentido de los otros, vecinos, es diferente al sentido hegemónico que tiene el "ser vecino" actualmente, el cual se caracteriza por discreto y distante, llegando a ser de ayuda extraordinaria activa, al mismo tiempo que hace gala de una discreción ordinaria permanente (Araujo \& Martuccelli, 2012), siendo éste sentido el que al parecer influye en la fase de recuperación, cuando aún los sujetos afectados habitan en las mediaguas, situación que produce irritaciones y conflictos. Estas acciones se condicen con el sentido de la amistad de la mayoría de los/as chilenos/as, o sea una relación de incondicionalidad, de entrega y de compromiso, tanto en los buenos momentos como en los momentos de desgracia (Araujo \& Martuccelli, 2012).

"el barrio se unió en ese momento, se juntó, nos sentamos [...] estábamos sentados al lado de las antenas de televisión [...] y se sienten los ruidos, me paré, y las luces".

(Mujer, 64 años, La Poza Centro)

"del segundo día estuve en la sede de la junta de vecinos, porque empezaron a llegar las ayudas [...] iba llegando y nosotros la íbamos distribuyendo [...] con una ficha del jefe de hogar, con su señora, y los hijos por edades [...] se lo entregué a una asistente social de la municipalidad [...] vinieron como a los quince días de la municipalidad [...] y dijo "así debimos hacerlo nosotros"”.".

(Hombre, 70 años, dirigente social, Mariscadero)

Solidaridad Nacionalista: Acciones orientadas a brindar ayuda humanitaria, sea esta material, emocional o espiritual a aquellos sujetos afectados por un desastre. La movilización de sujetos para ayudar a otros sujetos en contexto de desastre, se explica desde una característica intrínsecamente nacional, desde lo denominado por los sujetos como "chilenidad". Éstas fueron de vital importancia para los sujetos protagonistas del desastre, en el sentido de valorar la ayuda humanitaria brindada por los otros, similares a ellos, pero no afectados ni por el terremoto ni por el maremoto o tsunami, más que la ayuda de organizaciones humanitarias de la sociedad civil, o por 
las acciones desplegadas por el Estado, las cuales tuvieron un tiempo de tardanza mayor a las dos anteriores. Dicha solidaridad especialmente caracterizada en las clases vulnerables y en algunas capas medias se ha sedimentado como tradición en la cultura chilena hegemónica, invocada incluso en actividades solidarias televisadas para otros fines.

"[...] que cuando se trata de ayudar, los chilenos somos muy bondadosos, caritativos. $Y, y$, tenemos algo que no nos cabe en el corazón, adentro, ¿me entiende? Tenemos algo maravilloso".

(Hombre, 50 años, Mariscador, Mariscadero)

"mire le voy a explicar, la gente particular se portó muy bien con nosotros, porque al otro día la gente llegaba con sus camionetitas ahí y le traía cualquier cosita, le traía fruta le traía pancito, harina, fideos, todo eso traía la gente, al otro día siguiente al tiro, porque si hubiésemos esperado ayuda del gobierno [...] ¡nos habríamos muerto de hambre poh'! Y toda la gente estaba en las mismas condiciones".

(Hombre, 60 años, botero y sindicalista, La Poza)

En ese sentido, las narrativas de los sujetos que protagonizaron esta fase del desastre, dan cuenta de narrativas subalternas a las narrativas dominantes o hegemónicas con respecto al individuo producido actualmente en nuestra sociedad. Estas narrativas subalternas se presentan como acontecimientos extraordinarios (Goffman, 1974), desde donde pueden emerger nuevas narrativas liberadoras del etiquetamiento imperante sobre el tipo de individuo que se produce en el contexto social chileno actual. Por lo que como afirman Araujo y Martucelli (2012) no existe el homo neoliberal propiamente tal, sino que existe una narrativa hegemónica sobre el tipo de individuo a producir por el sistema capitalista neoliberal chileno, el cual se orienta por la competencia y la maximización de ganancias y triunfos individuales por sobre los demás. En ese sentido, corresponde a un tipo ideal de individuo para el sistema socioeconómico hegemónico, pero no necesariamente a una totalización del sujeto chileno. El desastre en tanto se abre como una ventana en donde emerge y se articula una narrativa que caracteriza otro tipo de individuo, que se diluye en del devenir del tiempo posterior al incidente crítico básicamente a raíz del encuadre del contexto social, político y económico presentado como pruebas societales para los sujetos, como lo es por ejemplo el acceso a la vivienda durante la reconstrucción, pues se promociona el acceso a la vivienda, individualizado en familias y no en barrios generando una red comunitaria en un territorio acotado.

Se destaca además los saqueos como un comportamiento orientado primeramente a la sobreviviencia, sin embargo contienen una carga significativa mayor. Desde las ciencias sociales se advirtió acerca de una destrucción significativa del tejido social y del "avance correlativo de la búsqueda egocéntrica del éxito y de la posesión material, vale decir una especie de repliegue individualista y narcisista acompañado de un curioso imaginario de prescindencia de lo social" (Baeza, 2010, p.57). Los saqueos se entienden como anomia, cuando los actores tienen estructuralmente anhelos que la sociedad es incapaz de satisfacer (Durkheim, 2004), explicarlos bajo la tesis de que la cultura engendra deseos que, inscribiéndose como expectativas en los individuos, instaura una distancia social y una frustración a veces generalizada en situaciones sociales incapaces de satisfacerlas (Bourdieu, 1999), en este caso los deseos que engendra la cultura de consumo promovida por el sistema capitalista neoliberal. Se pueden comprender como un momento de "pasar la cuenta" al sistema de desigualdad existente en Chile (Garcés, 2010), o un momento de tener lo que de otra forma no se había podido adquirir (Rojo, 2010). No obstante, yendo más allá, las narrativas dan cuenta que además estas estrategias se vinculan con el contexto situado en donde suceden. En otras palabras, ocurren dentro de un contexto de crisis de gobernabilidad (Baeza, 2010). Siguiendo la misma línea, se cumple la tesis de que "en situación de crisis aguda, las subjetividades sociales operaron con un grado de libertad inesperado, suscitando distintas motivaciones, todas ellas legitimadas 0 , por el contrario, contenidas según códigos de conducta muy individuales" (Baeza, 2010, p.64). Sumado a lo anterior, el Estado no solo no logra cumplir su rol de garante de la protección civil durante el terremoto y el tsunami, sino que tampoco su rol de garante de la seguridad ciudadana. Pese a esto, y como figura en las narrativas, el Estado pone mayor énfasis en la seguridad ciudadana que en la misma protección civil en el contexto del desastre específicamente cuando se declara estado de sitio. En ese sentido, el centralismo del Estado chileno (Garcés, 2010) y la figura del Estado portaliano (Góngora, 1981; Salazar, 1985) se convierten en un factor de vulnerabilidad ante el riesgo de desastre por un lado, y además en 
un factor de vulnerabilidad en el sentido de la seguridad ciudadana, razón que explica tanto la incapacidad de los gobiernos locales de resguardar la seguridad en el contexto del desastre, y por otro lado la necesidad de la presencia de los militares sostenida por la ciudadanía.

"también nos metimos a saquear [...] nosotros saqueamos en el Líder y en el Unimarc [...] el 27 [...] en el día [...] temprano quedó la embarrá [...] todos, gente que no perdió ni una cuchara del cerro Alto, camionetá de cuestiones, iban y venían con las camionetá de víveres [...] yo solo saquié [...] entré a saquear porque no había nah pa comer [...] era algo pa comer [...] cuando ví también entré [...] esos con camionetá estuvieron vendiendo después [...] yo saqué leche, si llegué casi al último [...] saqué esta cuestión de cervecero [...] redondos [...] de pavo, saqué unos seis, saqué unos jamones de praga, leche [...] hasta comida pal perro saqué $[\ldots]$ justo pillé una cajita, una cajita de esas donde vienen yogures [...] estaban todos sacando [...] los carabineros estaban viéndolos, [...] le dijeron a un cabro: "¿te vai a comer un computador?" [...] carabineros estaba mirando, no hacían nada, dejaban [...] estaban paraos mirando $[\ldots]^{\prime \prime}$.

(Hombre, 54 años, pescador, La Poza)

Existen diferencias con respecto al sentido que tuvo el saqueo: Los individuos de clases sociales vulnerables: Obreros no calificados del rubro pesquero, obreros no calificados del rubro de los servicios y en situación de indigencia, identificaron el saqueo como un acto de sobrevivencia en el caso de la extracción de bienes que servían para dicho cometido: Leche, pañales, alimentos en conserva, harina, entre otros; y como un acto delictivo, de vandalismo y por tanto reprochable. Por otro lado aquellos de clases medias: Obreros profesionales y pequeños propietarios de empresas familiares (pequeña burguesía local), significaron el saqueo, sin distinción como un acto de vandalismo, delictivo y por lo tanto reprochable, sumado al temor que sintieron antes de la llegada del Ejército de que sufrieran saqueos en sus propiedades a manos de "los otros", externos a su grupo primario de apoyo, familiar o amical. La otra diferencia de significación tiene que ver con el contexto en donde se produjo el saqueo: La sustracción de bienes de locales comerciales por un lado y la sustracción de bienes de la costa los tres días posteriores al tsunami, siendo el segundo, identificado como un tipo de saqueo al entender que lo que se encontraba en la playa era propiedad de quienes habitaban a orillas de ella. Al respecto, nuevamente emerge la figura de "los otros", provenientes de los cerros quienes saquean las playas. Se abre un debate con dos aristas al respecto y que tiene relación con la perspectiva moral desde donde significan las tácticas de sobrevivencia ejecutadas por los sujetos, y por otro lado, del concepto y del valor de la propiedad privada, según el tiempo y espacio donde ésta se sitúe.

"Los helicópteros que andaban. 0 sea, la Presidenta venía llegando y [...] el pueblo robaban, gritaban, la gente alerta y hacía que creían que venía la ola y se metían a robar. El pueblo, olvídalo [...] hay un señor que disparó al aire, tratando de proteger su negocio. Venia esta turba así, avanzando, avanzando, y él disparó, disparó, disparó, y siguieron avanzando así, na [...] y finalmente se sentó y dijo: "ya po, saquen todo lo que quiera". No podía hacer nada, no podía hacer nada (con rabia y asombro, a la vez). Estaban como, no sé. Peor que ratas".

(Mujer, 50 años, microempresaria, El Cable)

Otras de las acciones relevantes relatadas en las narrativas son aquellas orientadas a la búsqueda de ayuda humanitaria. Al respecto existen detalles relevantes de mencionar. Primero, que para el caso de los sujetos de clase media afectados por el desastre sus acciones tuvieron orientadas a la búsqueda de ayuda entre su grupo familiar y/o grupo amical. Existe una clara distinción en este acto, al no vincularse con los sujetos de clases vulnerables que recibieron ayuda, identificados como 'los otros' - potencialmente peligrosos-, buscando organizaciones no gubernamentales distintas a las que ayudaron a aquel grupo. El acceso a la ayuda humanitaria se podría clasificar como un tipo de distinción de clase social (Bourdieu, 1999). En el caso de la fase de recuperación, este tipo de sujetos no tuvieron acceso a subsidios de reconstrucción, por lo que el acceso a capital lo consiguieron a través de créditos bancarios. Esto se debe al rol subsidiario que juega el Estado en nuestro país, lo que significa que focaliza en crear acceso a oportunidades a las clases identificadas como en vulnerabilidad social. Para el caso de los sujetos de clases vulnerables éstos tuvieron acceso a subsidios para la reconstrucción de sus viviendas, ayuda humanitaria entre sus familiares y posteriormente sus amistades. Desde el mismo 27 de febrero comenzaron a recibir ayuda de otros sujetos de forma particular que provenían de otras comunas del país. Este tipo de sujetos recibió todo tipo de ayudas posteriormente, tanto del sector público como del sector privado, generando conflictos entre los vecinos/as. 
Como efecto de la distinción en el acceso a opor tunidades para la recuperación y reconstrucción, se produjeron conflictos vecinales en torno a la competencia por el acceso a la ayuda humanitaria, especialmente el acceso a bienes como alimentos, provocando la acumulación desmedida de parte de algunas familias y la emergencia de caudillismos con el objeto de beneficiar primero a la familia y los/as amigos/as Este tipo de situaciones se debe principalmente a la ausencia de sentido de comunidad, y a la individualización que se realiza al momento de por un lado, recurrir a la ayuda a humanitaria, y por otro lado la forma en que las instituciones regulan esta ayuda y/o la otorgan.

"Pero después de esto, como le digo, la gente se inhumanizó, porque en vez de unirse la gente, esto como que las desunió porque cada uno quería recibir más y no le importaba si la vecina de al lado recibía algo".

(Mujer, 75 años, Mariscadero)

\section{Recuperación}

La recuperación se identifica principalmente con la reconstrucción. De hecho, el concepto de recuperación es ajeno a los sujetos, utilizando el término "reconstrucción" al referirse a todo el proceso de recuperación posterior al incidente crítico. El foco se centra en el acceso a bienes y servicios, en especial alimentos, medicamentos y mediaguas o refugios, como también el acceso a subsidios de reparación y de acceso a viviendas. No obstante existe una clara necesidad de que el Estado no solo sea garante de las reconstrucción de lo material, sino que también lo sea de la recuperación emocional y psicosocial de los/as afectados por el desastre. En el sentido emocional, respecto a la atención psicológica a trastornos de la esfera de la ansiedad como lo es el Trastorno de Estrés Postraumático, y en el sentido psicosocial, respecto a la regeneración del capital social entendida como materia prima para el desarrollo de futuras estrategias comunitarias para la reducción del riesgo de desastres, pues la competencia por la ayuda humanitaria y la atomización familiar para el acceso a subsidios para la reconstrucción genera conflictos en la comunidad, indebidamente mediados o no resueltos. Existe una valoración del apoyo y contención emocional durante las primeras semanas posteriores al incidente crítico, sin embargo se enfatiza en la necesidad de que dicho apoyo sea continuo y programático durante el tiempo necesario para la restitución de la estabilidad emocional.

"hubo una señora que no sé de a dónde era, que decía que era psicóloga que vino después y todo el asunto, que en realidad nos trataba, nos ayudaba bastante, era bien cariñosa. Era como pa, pa levantarnos el ánimo, ella trataba de decir que teníamos que pararnos, que, tenemos que salir adelante".

(Hombre, mariscador, 50 años, Mariscadero)

"ojalá las autoridades se hagan cargo, creen cultura, incluyan en las materias, que haya mejor ayuda [...] que tomen más cartas en el asunto en los tsunamis en cómo reaccionar la gente, hay gente que han pasado 4 años y aún no tienen la casa que le prometieron [...] cuatro años, suficiente tiempo como para tenerle una solución a alguien [...] enfocarse en planes de emergencias [...] y después lo que sigue [...] apoyo psicológicos [...] hay profesionales [...] hay cómo estudiar [...] salí endeudado, pero sales con título para trabajar y aportar a la sociedad [...]".

(Mujer, 19 años, voluntaria de ONG, El Cable).

El fin de las acciones y estrategias desplegadas fue el restablecimiento de la cotidianeidad. A su vez se entiende que éstas fueron de cierta manera condicionadas por las oportunidades según la posición dentro de la estructura de clases de los sujetos.

\section{Factores de vulnerabilidad}

Se identifican tres tipos de factores de vulnerabilidad ante el riesgo de desastres, los cuales articulados consisten en el trasfondo semiótico-material desde donde se construye el significado y el sentido del desastre, en los distintos niveles sistémicos presentes en las narrativas de las experiencias de quienes protagonizaron el desastre:

- Factores Político-Institucionales: los cuales para el caso de esta investigación tienen relación con la política pública deficitaria en materia de protección civil en Chile. Actualmente, el modelo de un Estado céntrico, restando poder y toma de decisiones a los gobiernos locales, lo que provoca probablemente la focalización en la seguridad ciudadana en desmedro de la protección civil en contexto de desastre. 
- Factores Socioeconómicos: El desastre afectó principalmente a individuos de clases vulnerables, algo similar a la mayoría de los desastres anteriormente estudiados (Lawell, 2004). No obstante, la diferenciación de clases sociales deviene en factor de vulnerabilidad al contar con un Estado subsidiario que focaliza sus políticas de protección social en las clases vulnerables en desmedro de la clases medias, quienes también fueron afectadas por el desastre y quedaron en una situación de desamparo frente al Estado y forzándolos a recurrir a la oferta presente en el mercado para consolidar su recuperación, ya sea material como emocional.

- Factores Psicosociales: El tipo de individuo producido en Chile actualmente está focalizado en primero que todo articular su propia experiencia de vida (Dubet, 2010), y en superar todas las pruebas societales que se presentan en su trayectoria de vida (Araujo \& Martuccelli, 2012), ensimismado por el consumo y el exitismo, en desmedro de un sentir colectivo más allá del núcleo familiar, sino en la promoción de comunidad. Al existir redes sociales débiles, y casi ausencia de comunidad, existe menos capital social en tanto, menos probabilidades de generar comunidades resilientes para enfrentar nuevos desastres.

\section{REFLEXIONES FINALES}

Los desastres corresponden a un punto de inflexión en las tramas históricas de diferentes actores: Individuos, familias y comunidades. A su vez estas tramas están situadas en un territorio o contexto en particular. Los desastres, se posibilitan como tales desde un trasfondo semiótico-material desde donde se conjugan relaciones de poder, tradiciones, conocimientos, articulándose en una nueva realidad social. Desde esta perspectiva, los desastres no son naturales, son socionaturales. A su vez, se identifica, a través de las estrategias de afrontamiento posterior al incidente crítico, relatadas como narrativas subalternas a la narrativa hegemónica que sustenta el proceso de individuación presente, un sujeto como actor social, o sea, con la capacidad de transformar su realidad social y política en tanto afecta las relaciones de poder y de dominación.

En este sentido, los desastres se convierten en una situación propicia para comprender a una sociedad o una comunidad en particular, considerando y relevando su capacidad de resiliencia y el fortalecimiento de su capital social, particularmente a través de los procesos de significación y de sentido que otorgan lo sujetos a su experiencia en el desastre, desde donde emergen narrativas alternativas que se puede visualizar y construir el sujeto como actor social en desmedro del sujeto hiperindividualizado y condenado a la superación de pruebas societales, promovido por la sociedad chilena actual. Pues producir resiliencia requiere algo más que reducir la vulnerabilidad: hace falta empoderar a los individuos y disponer de unas sólidas instituciones sociales y estatales que puedan ayudar a las personas a hacer frente a los acontecimientos adversos. Debe existir primero una concientización y una autoidentificación como comunidad resiliente para lograr fortalecer el capital social, en tanto se narrativiza en acto de producción, un tipo diferente de individuo. Este camino significa que los aprendizajes descritos por los sujetos a través de sus narrativas, más allá de aquellos referentes al autocuidado ya promovido por el Estado, pueden centrarse en la promoción de la importancia de generar una narrativa sobre un "nosotros" comunitario.

Considerando esta premisa, sería posible abrir o profundizar ciertas líneas de investigación con dos compromisos fundamentales: por un lado, producir conocimiento para mejorar la calidad de vida de los sujetos chilenos, siendo parte de las estrategias de reducción del riesgo de desastres, y por otro lado, producir conocimiento situado, local, que ponga a los sujetos y las comunidades como centro de la reducción del riesgo de desastres, en diferentes niveles territoriales, incorporando la subjetividad de la experiencia en desastres, con una postura epistemológica, política y ética, que releve el modelo político, el modelo socioeconómico y el trasfondo cultural como factores que condicionan de cierta forma el comportamiento de los sujetos en estos contextos. Estas líneas de investigación podrían además desarrollarse desde una sociología de los desastres o desde una psicología social de los desastres, la cual se interese por los procesos de individuación y de subjetivación y su relación con el comportamiento durante el proceso del riesgo de los desastres.

\section{REFERENCIAS}

Araujo, K. \& Martucelli, D. (2012). Desafíos Comunes. Retrato de la sociedad chilena y sus individuos. Santiago de Chile: LOM Ediciones

Ávila, M. (2010). Biopolítica: Neoliberalismo y Subjetividad. En Revista Paralaje, Dossier Biopolítica y amenaza: a seguridad sobre la vida. Valparaiso: Pontificia Universidad Católica de Valparaíso. 
Baeza, M. (2010). Carnaval perverso: Terremoto + tsunami y saqueos en el Chile de 2010. Revista Sociedad Hoy, 19, 53-69.

Balasch, M. \& Montenegro, M. (2003). Una propuesta metodológica desde la epistemología de los conocimientos situados: Las producciones narrativas. Encuentros en Psicología Social, 1(3), 44-48.

Bauer, M. (1996). The narrative interview: comment s on a technique of qualitative data collection. Papers in Social Research Methods, - Qualitative Series, Vol. 1. London: London School of Economics, Methodology Institute.

Beck, U. \& Beck-Gernsheim, E. (2003). La individualización. El individualismo institucionalizado y sus consecuencias sociales y políticas. Madrid: Editorial Paidós,

Bernasconi, O. (2011). Aproximación narrativa al estudio de fenómenos sociales: principales líneas de desarrollo. Acta Sociológica, 56(3), 9-36. DOI: http://dx.doi.org/10.22201/ fcpys.24484938e.2011.56.28611

Biglia, B \& Bonet-Martí, J (2009). La construcción de narrativas como método de investigación psicosocial. Prácticas de escritura compartida. Forum: Qualitative Social Research Sozialforchung, 10(1). DOI: http://dx.doi.org/10.17169/fqs-10.1.1225

Bourdieu, P. (1991). El sentido práctico. Madrid: Editorial Taurus.

Bourdieu, P. (1999) La distinción. Criterio y bases sociales del gusto. Madrid: Editorial Taurus Ediciones.

Bogdan, R. \& Taylor, S.J. (1987). Introducción a los métodos cualitativos de investigación. La búsqueda de significados. Madrid: Paidós.

Bruner, J. (1991). Actos de Significado. Más allá de la Revolución Cognitiva. Madrid: Alianza Editorial.

Campos, A. (1999). Educación y prevención de desastres. San Jose, Costa Rica: UNICEF, FLACSO, La RED.

Canales, M. (2006). Metodologías de Investigación Social. Introducción a los oficios. Santiago de Chile: Lom Ediciones.

Chardon, A. (2008). Amenaza, vulnerabilidad y sociedades urbanas. Una reflexión desde la dimensión institucional. Gestión y Ambiente: Seccional Medellín, 11(1), 28-46.

Cottet, P. (2013). Tres versiones del diseño para investigaciones sociales. En Canales, M. (Ed.), Investigación Social. Lenguajes del Diseño. Santiago de Chile: Lom Ediciones, pp. 13-42.

Dresdner, J. \& Sehnbruch, K. (2010). El impacto del sismo 2010 sobre el mercado laboral de la Región del Bíobío. Revista Sociedad Hoy, 19, 71-96.

Dubet, F. (2010). Sociología de la Experiencia. Madrid: Editorial Complutense.

Durkheim, E. (2004). El Suicidio. Estudio de sociología. Buenos Aires: Editorial Losada.

Foucault, M. (2004). Seguridad, territorio y población. Buenos Aires: Fondo de Cultura Económica.

Garcés, M. (2010). Terremoto natural y terremoto social en Chile. En Aguilera, S. (Ed.), El Terremoto social del Bicentenario. Santiago de Chile: Lom Ediciones, pp. 67-86.

Geertz, C. (1983). Conocimiento Local. Ensayos sobre la interpretación de las culturas. Madrid: Editorial Paidós.

Goffman, E. (1974). Estigma. La identidad deteriorada. Buenos Aires: Amorrortu Editores.

Góngora, M. (1981) Ensayo Histórico sobre la Noción de Estado en Chile en los Siglos XIX y XX. Santiago de Chile: Ediciones de la Ciudad.

Haraway, D. (1995) Ciencia, cyborgs y mujeres. La reinvención de la naturaleza. Madrid: Cátedra.

Instituto Nacional de Estadísticas INE. (2017). Censo 2017. INE [Web]. Recuperado de https://www. censo2017.cl/ [31 Jun. 2019]

Lavell, A. (2004). Los conceptos, estudios y práctica en torno al tema de los riesgos y desastres en América Latina: Evolución y cambio, 1980-2004: El rol de La Red, sus miembros y sus instituciones de apoyo. San José, Costa Rica: Facultad Latinoamericana de Ciencias Sociales, FLACSO.

Narváez, L., Lavell, A. \& Pérez, G. (2009). La Gestión del Riesgo de Desastres. Un enfoque basado en procesos. Proyecto Apoyo a la Prevención de Desastres en la ComunidadAndina. Lima: PREDECAN.

Ricoeur, P. (2008) Tiempo y Narración. Ciudad de México: Siglo XXI.

Riessman, C. (2001). Analysis of personal narratives. En J. Gubrium E J. Holstein (Eds.), The SAGE

Handbook of Interview Research: The Complexity of the Craft. Thousand Oaks, CA.: SAGE

Publications. 
Riessman, C. (2008). Narrative Methods for the Human Sciences. London: SAGE Publications.

Rojas, J. (2010). Vulnerabilidad social, neoliberalismo y desastre: sueños y temores de la comunidad desplazada/damnificada por el terremoto/tsunami. Revista Sociedad Hoy, 19, 113-140.

Rojo, G. (2010). Saqueos. En Aguilera, S. (Ed.), El Terremoto social del Bicentenario. Santiago de Chile: Lom Ediciones, pp. 103-114.

Romero, H. \& Vidal, C. (2015). Exposición, Sensibilidad y Resiliencia ante los desastres de las Ciudades de Concepción-Talcahuano, Chile Central. En CIVDES (Ed.), Vulnerabilidades y Desastres Socionaturales: Experiencias recientes en Chile. Santiago de Chile: Centro de Investigación en Vulnerabilidades y Desastres Socionaturales (CIVDES), pp. 12-27.

Ruiz, J. (2003). Metodología de la Investigación Cualitativa (zera ed). Bilbao: Universidad de Deusto.

Ruiz, C. \& Boccardo, G. (2014). Los Chilenos bajo el Neoliberalismo. Clases y conflicto social. Santiago de Chile: Ediciones y publicaciones El Buen Aire S.A.

Salazar, G. (1985). Labradores, peones y proletarios. Formación y crisis de la sociedad popular chilena del siglo XIX. Santiago de Chile: Ediciones Sur.

Ugarte, A. M. \& Salgado, M. (2014). Sujetos en emergencia: Acciones colectivas de resistencia y enfrentamiento del riesgo ante desastres; el caso de Chaitén, Chile. Revista INVI, 29, 143-168.

Sandoval, J. (2004). Representación, discursividad y acción situada. Introducción crítica a la psicología social del conocimiento. Santiago de Chile: Editorial Universidad de Valparaíso.

Sandoval, J. (2013). Una perspectiva situada de la investigación cualitativa en ciencias sociales. Cinta de Moebio: Revista de Epistemología de Ciencias Sociales, 46, 37-46. DOI: http://doi.org/10.4067/ So717-554X2013000100004

Valenzuela, K. (2010). ¿La vuelta de los sin techo? Análisis de la acción colectiva desplegada en campamentos de emergencia tras el terremoto del 27/F. Revista Sociedad Hoy, 19, 141-152. 